\title{
Liquid crystal phase and waterlike anomalies in a core-softened shoulder-dumbbells system
}

Cite as: J. Chem. Phys. 132, 164505 (2010); https://doi.org/10.1063/1.3386384

Submitted: 14 December 2009 . Accepted: 18 March 2010 . Published Online: 26 April 2010

Alan Barros de Oliveira, Eduardo B. Neves, Cristina Gavazzoni, Juliana Z. Paukowski, Paulo A. Netz, and Marcia C. Barbosa

\section{ARTICLES YOU MAY BE INTERESTED IN}

Comparison of simple potential functions for simulating liquid water

The Journal of Chemical Physics 79, 926 (1983); https://doi.org/10.1063/1.445869

Structure and mobility of water confined in $\mathrm{AlPO}_{4^{-5}}$ - nanotubes

The Journal of Chemical Physics 146, 234509 (2017); https://doi.org/10.1063/1.4985626

Perspective: Surface freezing in water: A nexus of experiments and simulations

The Journal of Chemical Physics 147, 060901 (2017); https://doi.org/10.1063/1.4985879

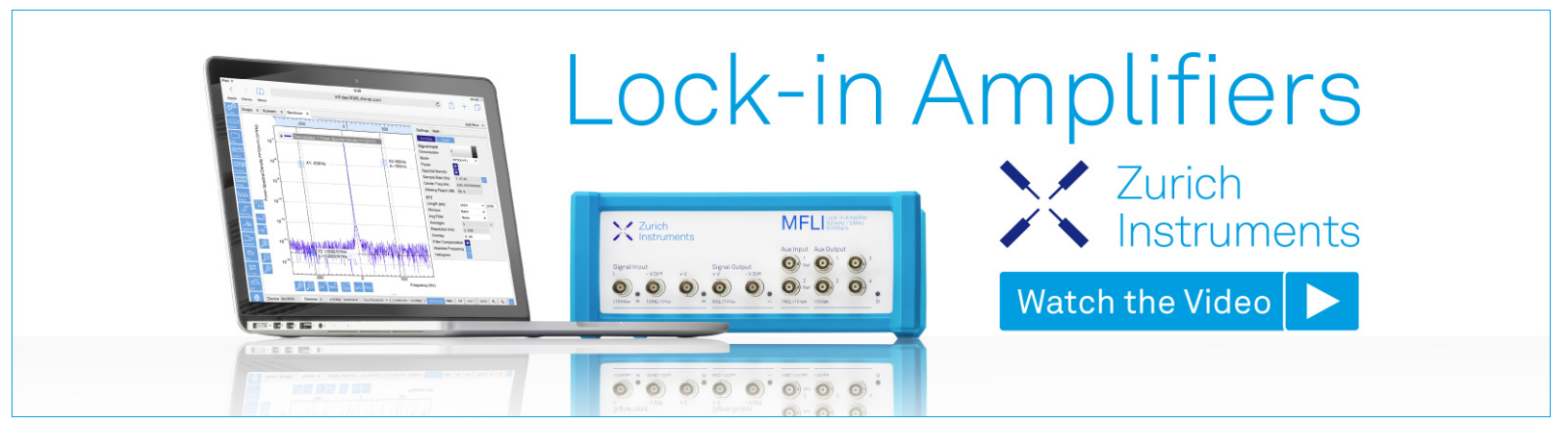

J. Chem. Phys. 132, 164505 (2010); https://doi.org/10.1063/1.3386384

132,164505

(C) 2010 American Institute of Physics. 


\title{
Liquid crystal phase and waterlike anomalies in a core-softened shoulder-dumbbells system
}

\author{
Alan Barros de Oliveira, ${ }^{1}$ Eduardo B. Neves, ${ }^{2}$ Cristina Gavazzoni, ${ }^{3}$ Juliana Z. Paukowski, ${ }^{3}$ \\ Paulo A. Netz, ${ }^{4}$ and Marcia C. Barbosa ${ }^{3, a)}$ \\ ${ }^{1}$ Departamento de Física, Universidade Federal de Ouro Preto, Ouro Preto, MG 35400-000, Brazil \\ ${ }^{2}$ Petrobrás, Av. Elias Agostinho, 665, OP-EN-Imbetiba, Macaé, RJ 27913-350, Brazil \\ ${ }^{3}$ Instituto de Física, Universidade Federal do Rio Grande do Sul, Porto Alegre, RS 91501-970, Brazil \\ ${ }^{4}$ Instituto de Química, Universidade Federal do Rio Grande do Sul, Porto Alegre, RS 91501-970, Brazil
}

(Received 14 December 2009; accepted 18 March 2010; published online 26 April 2010)

\begin{abstract}
Using molecular dynamics we investigate the thermodynamics, dynamics, and structure of 250 diatomic molecules interacting by a core-softened potential. This system exhibits thermodynamic, dynamic, and structural anomalies: a maximum in density-temperature plane at constant pressure and maximum and minimum points in the diffusivity and translational order parameter against density at constant temperature. Starting with very dense systems and decreasing density the mobility at low temperatures first increases, reaches a maximum, then decreases, reaches a minimum and finally increases. In the pressure-temperature phase diagram the line of maximum translational order parameter is located outside the line of diffusivity extrema that is enclosing the temperature of maximum density line. We compare our results with the monomeric system showing that the anisotropy due to the dumbbell leads to a much larger solid phase and to the appearance of a liquid crystal phase. ( 2010 American Institute of Physics. [doi:10.1063/1.3386384]
\end{abstract}

\section{INTRODUCTION}

Several anomalies present in liquid water are believed to be due to the formation and disruption of hydrogen bonds. Specific angles and distances between water molecules are necessary to form such bonds, which lead to a dramatic competition between open low-density and closed high-density structures, depending on the thermodynamic state of the liquid. Although in real water this competition actually acts in the second neighbors distances, ${ }^{1}$ it could be modeled in a "supermolecule" approach as a competition between two preferred interparticle distances in a spherically symmetrical intermolecular interaction potential. Based on this mechanism, isotropic systems where particles interact through core-softened potentials were considered for studying the odd behavior of water. ${ }^{2-27}$

One of the biggest challenges for adopting these models to describe water and systems that exhibit the anomalies present in water is to reproduce some aspects of the anomalous behavior, ${ }^{28,29}$ such as the existence of a temperature of maximum density (TMD), liquid-liquid phase transition or nonmonotonic diffusion behavior with respect to isothermal pressure variation. ${ }^{30-32}$

Water, however, is not an isolated case. Many materials exhibit the anomalies present in water. For example, density anomaly was found experimentally in $\mathrm{Se}_{x} \mathrm{Te}_{1-x},{ }^{33}$ and $\mathrm{Ge}_{15} \mathrm{Te}_{85}{ }^{34}$ Liquid sulfur displays a sharp minimum in the density, ${ }^{35}$ related to a polymerization transition. ${ }^{36}$ Waterlike anomalies were also found in simulations for silica, ${ }^{37-41}$ silicon, ${ }^{42}$ and $\mathrm{BeF}_{2} \cdot{ }^{38,43,44}$

${ }^{a)}$ Electronic mail: marcia.barbosa@ufrgs.br.
The recently proposed core-softened shoulder potential ${ }^{17-19,45}$ reproduces the density and diffusion anomalies. This potential can represent in an effective and orientation-averaged way the interactions between water pentamers characterized by the presence of two structuresone open and one closed-discussed above. Similarly, the thermodynamic and dynamic anomalies result from the competition between the two length scales associated with the open and closed structures. The open structure is favored by low pressures and the closed structure is favored by high pressures, but only becomes accessible at sufficiently high temperature.

Simple pair potentials are particularly interesting because they are computationally cheaper than molecular models as well as amenable under analytical treatments. ${ }^{18,46,47}$ For example, the recent perturbation theory developed by Zhou $^{48-50}$ is highly promising on attacking these sort of potentials.

In spite of core-softened potentials have been mainly used for modeling water, ${ }^{9,18,19,23,27,51-54}$ many other materials present the so-called waterlike anomaly behavior. In this sense, it is reasonable to use core-softened potentials as the building blocks of a broader class of materials which we can classify as anomalous fluids. This would allow us to fabricate anomalous liquids by imposing interparticle core-softened potentials. For instance, one could build a polymer that diffuses faster under higher pressures what would be a quite interesting property for manufacturing plastic materials. But polymers are anisotropic systems and up to now the literature on core-softened potentials leading to waterlike anomalies is restricted to spherical symmetric systems. 


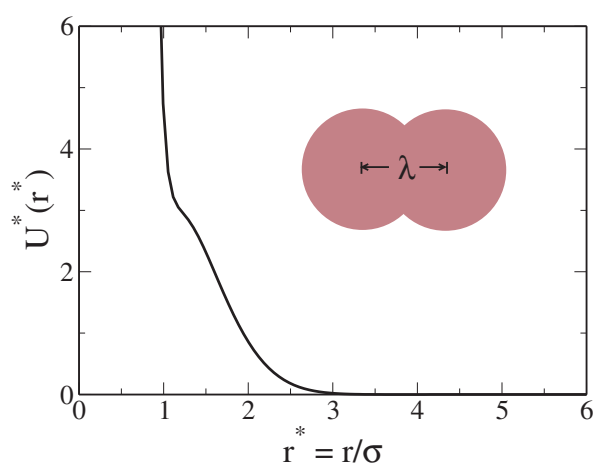

FIG. 1. Effective potential vs distance in reduced units.

Would the addition of an anisotropy in one direction to a system of particles interacting through a core-softened potential still maintain the anomalies present in the spherical symmetric system? Which new features the anisotropic system would exhibit? Here we address these questions by studying the pressure-temperature phase diagram of a model system made by rigid dimers. Each particle in the dimer interacts with the particles in the other dimers through a core-softened potential. The distance between the particles in the same dimer is chosen to be smaller than diameter in order to test the effect of introducing the anisotropy without disturbing the two other interaction scales.

The results obtained using the dumbbell system are compared with the pressure-temperature phase diagram of the monomeric system interacting by the same core-softened potential employed in the dimeric case.

Obviously anisotropic systems are not only computationally more complicated but also the addition of an extra degree of freedom yields a richer phase diagram. For instance, diatomic particles interacting through a Lennard-Jones potential ${ }^{55}$ exhibit a solid phase that occupies higher pressures and temperatures in the pressure-temperature phase diagram. In the case of the solid phase, the diatomic particles exhibit two close-packed arrangements instead of one observed in the monoatomic Lennard-Jones. ${ }^{56}$ Therefore, we also expect the dimeric system interacting through two length scales potential might have a phase diagram with a larger solid phase region in the pressure-temperature phase diagram than the one occupied by the solid phase in the monomeric system. Since in many cases the anomalies are close to the solid-liquid phase transition ${ }^{21,23}$ the dumbbell model studied in this paper might have the anomalous region in the pressure-temperature phase diagram located inside the solid phases region. We shall check if this is the case in this paper.

The remaining of this manuscript goes as follows. In Sec. II the model is introduced and the simulation details are presented. In Sec. III the results are shown and conclusions are found in Sec. IV.

\section{THE MODEL}

The model consists of $N / 2$ dimeric molecules (dumbbells) formed each by two spherical particles of diameter $\sigma$, linked rigidly in pairs with the distance $\lambda$ between their centers of mass as depicted in Fig. 1. Each particle within a dimer interacts with all particles belonging to other dimers with the intermolecular continuous shoulder potential ${ }^{18}$ given by

$$
U^{*}(r)=4\left[\left(\frac{\sigma}{r}\right)^{12}-\left(\frac{\sigma}{r}\right)^{6}\right]+a \exp \left[-\frac{1}{c^{2}}\left(\frac{r-r_{0}}{\sigma}\right)^{2}\right] .
$$

This potential can represent a whole family of two length scales intermolecular interactions, from a deep double wells potential ${ }^{17,45}$ to a repulsive shoulder, ${ }^{13}$ depending on the choice of the values of $a, r_{0}$, and $c$.

This potential for the monomeric system was studied with a very small attractive region, with $a=5, r_{0} / \sigma=0.7$, and $c=1$ so the liquid-gas unstable and metastable region would be avoided. ${ }^{18,19}$ This potential has two length scales within a repulsive ramp followed by a very small attractive well.

Here we explore the same interaction potential analyzed for the monomeric system, but for dumbbells. In particular we will assume $\lambda / \sigma=0.2$. In order to study the equilibrium pressure-temperature phase diagram, we use molecular dynamics simulations to obtain the pressure as a function of temperature along isochores, diffusion constant as a function of density and temperature and the behavior of the structure as a function of temperature and pressure.

We performed molecular dynamics simulations in the canonical ensemble using $N=500$ particles ( 250 dimers) in a cubic box of volume $V$ with periodic boundary conditions in the three directions, interacting with the intermolecular potential described above. The number density of the system is then $\rho=N / V$. The cutoff radius was set to $5.5 \sigma$. Pressure, temperature, density, and diffusion are calculated in dimensionless units

$$
\begin{aligned}
T^{*} & \equiv \frac{k_{B} T}{\epsilon}, \\
\rho^{*} & \equiv \rho \sigma^{3}, \\
P^{*} & \equiv \frac{P \sigma^{3}}{\epsilon}, \\
D^{*} & \equiv \frac{D(m / \epsilon)^{1 / 2}}{\sigma} .
\end{aligned}
$$

In some state points we also carried out simulations with the same model but with 1000 (500 dimers) and 2000 (1000 dimers) particles using the large-scale atomic/molecular massively parallel simulator ${ }^{57}$ with essentially the same results. Further simulation details are discussed elsewhere. ${ }^{18}$

Preliminary simulations showed that depending on the chosen temperature and density the system was in a fluid phase but became metastable with respect to the solid phase. In order to locate the phase boundary between the solid and the fluid phases, two sets of simulations were carried out, one beginning with the molecules in a ordered crystal structure and the other beginning with the molecules in a random, liquid, starting structure obtained from previous equilibrium simulations. Thermodynamic and dynamic properties were calculated over 700000 steps for the first set (and 900000 steps for the second set), previously equilibrated over 

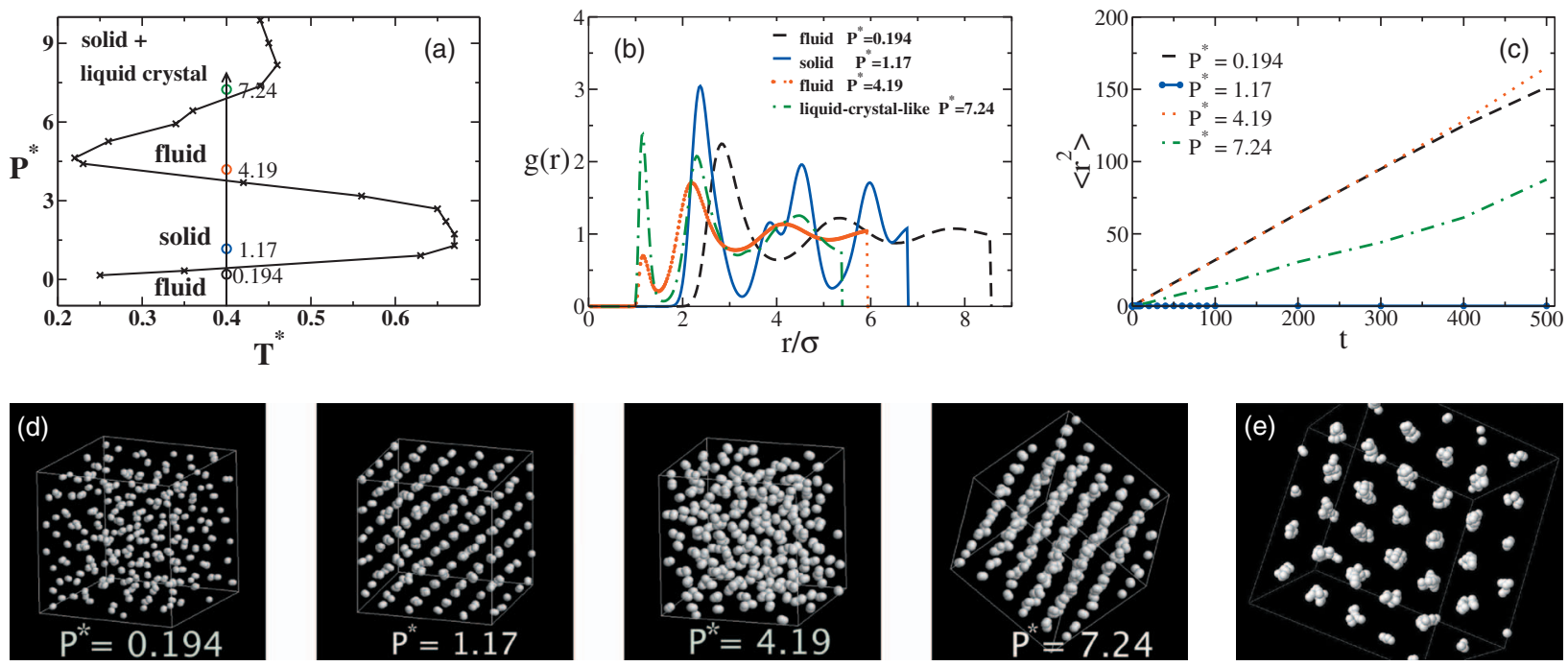

FIG. 2. (a) Reduced pressure vs reduced temperature phase diagram showing the liquid and solid phases. The arrow at $T^{*}=0.4$ crosses the regions with $P^{*}=0.194,1.17,4.19$, and 7.24 illustrated in the RDF. (b) RDF vs reduced distance for $T^{*}=0.4$ and $P^{*}=0.194,1.17,4.19$, and 7.24. (c) MSD vs time for the four regions illustrated in (a). (d) Snapshots of the configurations for $T^{*}=0.4$ and the same pressures as in panel (a). (e) A snapshot of the configuration for $T^{*}=0.4$ showing an ordering along lines for $P^{*}=7.24$.

200000 (or 300 000) steps. For the high pressures region the equilibration time was 500000 steps and the thermodynamic, dynamic, and structural properties were calculated over 2000000 steps. The time step was 0.001 in reduced units and the time constant of the Berendsen thermostat ${ }^{58}$ was 0.1 in reduced units. The internal bonds between the particles in each dimer remain fixed using the algorithm SHAKE (Ref. 59) algorithm, with a tolerance of $10^{-12}$.

The stability of the system was checked by analyzing the dependence of pressure on density and also by visual analysis of the final structure, searching for cavitation. The structure of the system was characterized using the intermolecular radial distribution function, $g(r)(\mathrm{RDF})$, which does not take into account the correlation between atoms belonging to the same molecule. The diffusion coefficient was calculated using the slope of the least square fit to the linear part of the mean square displacement, $\left\langle r^{2}(t)\right\rangle$ (MSD), averaged over different time origins. Both the $g(r)$ and $\left\langle r^{2}(t)\right\rangle$ were computed taking the origin as the center of mass of a dimer.

For analyzing the structure we define the structural anomaly region as the region where the translational order parameter $t$, given by

$$
t \equiv \int_{0}^{\xi_{c}}|g(\xi)-1| d \xi
$$

decreases upon increasing density. Here $\xi \equiv r \rho^{1 / 3}$ is the distance $r$ in units of the mean interparticle separation, computed by the center of mass of the dimers, $\rho^{-1 / 3}, \xi_{c}$ is the cutoff distance set to half of the simulation box times $\rho^{1 / 3}$, as in Ref. 19, $g(\xi)$ is the RDF as a function of the (reduced) distance $\xi$ from a reference particle. For an ideal gas $g=1$ and $t=0$. In the crystal phase $g \neq 1$ over long distances and $t$ is large.

\section{RESULTS}

\section{A. The phase diagram}

Figure 2 shows (a) the pressure-temperature phase diagram, (b) the RDFs, (c) the MSDs and, finally, (d) snapshots of the system at some relevant thermodynamic state points. The pressure-temperature phase diagram, illustrated in Fig. 2(a), displays at low temperatures a low density solid phase, a high density solid phase, a low density fluid phase, and a high density fluid phase. Near the boundaries of the high density solid phase, a liquid crystal-like (LCL) phase was also identified, as discussed below.

At intermediate temperatures, as the pressure is isothermally increased [following the arrow in the Fig. 2(a)] the system goes as follows: For low pressures the system is in the fluid phase, then as pressure increases it becomes solid, for higher pressures it becomes fluid again and then LCL for even higher pressures. For very high pressures the system becomes solid and for even more higher pressures it becomes fluid again (not shown in the figure).

The nature of the phases was investigated from three different ways: the RDF, Fig. 2(b), the MSD, Fig. 2(c), and the structural snapshots, Fig. 2(d). While for $P^{*}=1.17$ and 7.24, Fig. 2(d) shows ordered structures, for $P^{*}=0.194$ and 4.19 the structure is disordered, which could be typical for a liquid or a glass, depending on the mobility of particles in the system. According to the MSD illustrated in Fig. 2(c), for $P^{*}=0.194$ and $P^{*}=4.19$ particles show high mobility, whereas for $P^{*}=1.17$ particles do not move. This would be already expected from the corresponding RDF shown in Fig. 2(b). However, for $P^{*}=7.24$ particles move almost as fast as in a liquid phase, but the corresponding RDF and structural snapshots are rather solidlike.

Which type of phase could be present in $P^{*}=7.24$ ? In order to answer this question a movie with the evolution of the configurations was made. This movie (available in the website http://www.if.ufrgs.br/ barbosa/publication.html) 

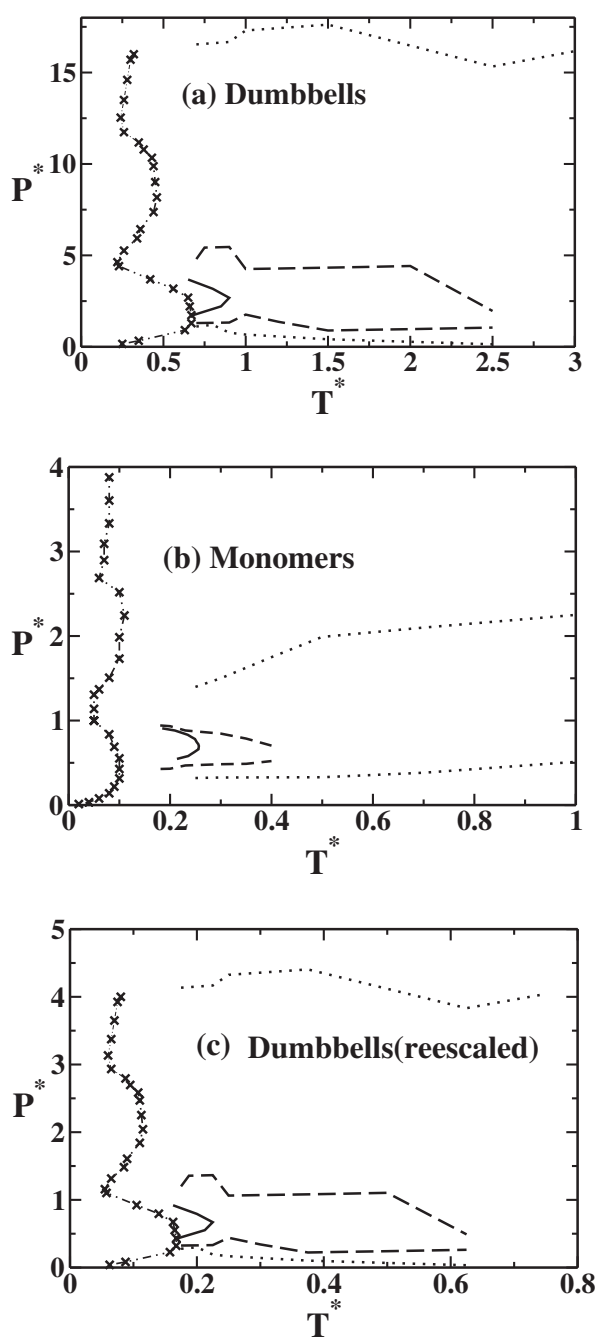

FIG. 3. Pressure vs temperature phase diagram for (a) dimeric and (b) monomeric particles interacting through the potential illustrated in Fig. 1, and (c) the rescaled dimeric results, in which pressure and temperature from (a) are divided by 4 (see the text for more details). The results shown in panel (b) were adapted from Refs. 18 and 19. The re-entrant line with crosses in (a) represent the boundary between the fluid and the solid phases. The bold solid, dashed, and dotted lines represent the TMD, the extrema, and the region of the structural anomaly, respectively, in both (a) and (b) panels.

shows that in this case particles actually move, but only in a stringlike fashion, which characterizes a LCL phase. Figure 2(e) shows that this phase is characterized by lines of dumbbell particles. The distance between the lines coincides with the minimum of the potential illustrated in Fig. 1 while the distance between the dumbbell particles along the same string is the shoulder distance in Fig. 1. This phase differs from the solidlike stripes observed in systems with competing interactions due to its mobility. ${ }^{60}$ Besides the presence of this LCL phase, the dumbbell system also exhibit a fluid phase at very high pressures. We could not yet identify clearly the phase boundary between the solid and the LCL phases, but work in this matter is under way.

The solid-liquid interfaces for (a) the dimeric and (b) the monomeric systems and (c) the dimeric rescaled by a factor of 4 (for a better comparison the monomeric case should be compared with the dimeric system with one fourth of the energy) are shown in Fig. 3 as crosses. Compared with the monomeric system, the solid phase occupies a larger region in the pressure-temperature phase diagram. This effect might be attributed to the dumbbells since the same is observed when the pressure-temperature phase diagram of a system made of dimeric Lennard-Jones particles is compared with monomeric Lennard-Jones particles. ${ }^{55,56}$ The effect of having a core-softened potential is not simply to increase the solid region but to give raise to a number of different solid phases. ${ }^{61}$

\section{B. The anomalies}

Similarly to the previously studied monomeric system ${ }^{18}$ anomalies were also found in its dumbbells version, considered in this work. We focused in the three anomalies already found in the monomers case, i.e., the density, diffusion, and structural anomalies, as described below.

(i) The density anomaly is the unusual expansion of the system upon cooling at constant pressure, a well-known effect which happens in water as discussed in the Sec. I. In NPT-constant ensemble this anomaly is characterized by a maximum of the density along isobars in the densitytemperature plane. Through thermodynamic relations ${ }^{62}$ we are able to equivalently detect this anomaly by searching for a minimum of the pressure along isochores in the pressuretemperature phase diagram. This was the technique used in this work since it is more suitable for the NVT ensemble.

The density anomaly region for both (a) dimeric and (b) monomeric systems are shown in Fig. 3 as solid bold lines. Both dimeric and monomeric systems have the usual noseshaped TMD line format, also found in molecular models for water $^{30,31}$ as well as for other isotropic potentials. ${ }^{22,51-53}$

For the dumbbells model the density anomaly region is located at higher pressures and temperatures and occupies a much larger region in the pressure-temperature phase diagram than that observed for the monomeric system. This can be evidenced by values for the maximum and minimum pressures and temperatures which enclose the TMD line for both systems, as shown in Table I.

(ii) We also studied the molecular mobility calculating the diffusion coefficient using the MSD averaged over different initial times

$$
\left\langle\Delta r(t)^{2}\right\rangle=\left\langle\left[r\left(t_{0}+t\right)-r\left(t_{0}\right)\right]^{2}\right\rangle .
$$

Then the diffusion coefficient is computed for the center of mass motion and is obtained from the relation

$$
D=\lim _{t \rightarrow \infty}\left\langle\Delta r(t)^{2}\right\rangle / 6 t .
$$

The usual procedure for detecting the diffusion anomalous region is to plot the diffusion coefficient versus density for fixed temperatures ${ }^{18,19,22,51,62}$ as depicted in Fig. 4. From this figure we see that for a certain range of densities the diffusion coefficient anomalously increases under increasing density, changing its slope from negative to positive into this range. This determines a local maxima and minima for the $D(\rho)$ plot. These extrema can be mapped into a pressuretemperature plane, determining the lines of extrema in the diffusion coefficient inside which particles move faster under compression or, equivalently, under increasing density. 
TABLE I. Maximum and minimum pressures and temperatures which bound (A) the TMD line (solid in Fig. 3) in both systems, (B) dynamic anomaly region (bounded by dashed lines in Fig. 3) for both systems, and (C) the structural anomaly region (bounded by dotted lines in Fig. 3) for both systems.

\begin{tabular}{|c|c|c|c|c|c|c|}
\hline & \multicolumn{2}{|c|}{ A } & \multicolumn{2}{|c|}{ B } & \multicolumn{2}{|c|}{$\mathrm{C}$} \\
\hline & Dimers & Monomers & Dimers & Monomers & Dimers & Monomers \\
\hline$P^{*} \max$ & 3.66 & 0.90 & 5.42 & 0.94 & 17.6 & 0.94 \\
\hline$P^{*} \min$. & 1.73 & 0.55 & 0.90 & 0.43 & 0.14 & 0.43 \\
\hline$T^{*} \max$ & 0.90 & 0.26 & 2.50 & 0.40 & $>3.0$ & 1.00 \\
\hline$T^{*} \min$ & 0.65 & 0.18 & 0.70 & 0.18 & $<0.70$ & 0.25 \\
\hline
\end{tabular}

Figure 3(a) illustrates the local diffusivity maxima and minima as dashed lines. Comparison between Figs. 3(a) and 3 (b) indicates that the diffusion anomaly region for dumbbells occupies a larger region in the $P-T$ plane than the diffusion anomaly region for the monomeric system. This can be evidenced by values for the maximum and minimum pressures and temperatures which enclose the diffusion anomalous region in Table I.

(iii) We also study the behavior of the translational order parameter. We see from Fig. 5 that $t$ decreases for increasing density in almost the entire range of densities, contrary of what is expected for a normal fluid. The local maximum/ minimum in the $t(\rho)$ plot can be mapped into a pressuretemperature plane (as discussed above) giving the boundary lines for the region of structural anomaly in the $P-T$ phase diagram, i.e., dotted lines in Fig. 3.

Comparison between Figs. 3(a) and 3(b) shows that the structural anomaly region is much broader for the dumbbells than for the monomers. It shall be stressed that the dumbbells system can be found in the liquid phase at pressures at high as $P^{*}=17.6$, which bounds the upper limit of the structural anomaly region. On the other hand, the lower limit for the structural anomaly region of the dumbbells model achieves pressures as low as 0.14 in reduced units, even lower than for the monomers case (which is 0.32 in reduced units).

The structural order parameter $t$ measures how ordered and disordered the system becomes with the increase in density. In the monomeric case, a region where the system becomes disordered as the density is increased is observed. This disorder is due to the presence of the two competing scales. As the density is increased a larger number of particles are observed in the two scales forming no particular structure and $t$ decreases with $\rho$. This, however, is limited by

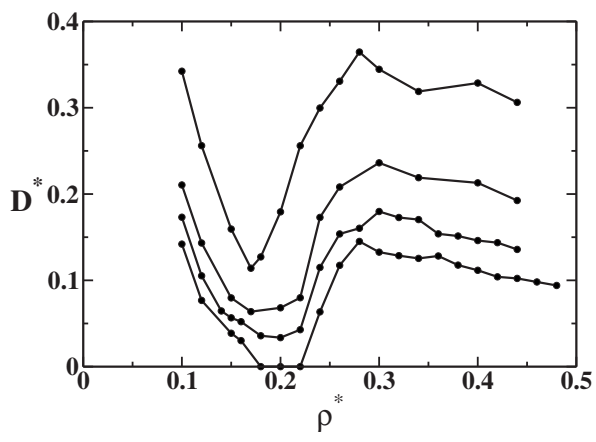

FIG. 4. Diffusion coefficient vs density at fixed temperatures, which are $T^{*}=0.65,0.75,0.90$, and 1.20 from bottom to top. a region of very high density, $\rho_{\text {tmax-mono }}$, where the system chooses the closest scale. In the case of the dimeric system the dimer can also order in different orientations increasing the possible arrangements. Consequently the density of maximum translational order parameter for the dimeric case is larger than the density of maximum $t$ for the monomeric case, $\rho_{\text {tmax-dimer }}>\rho_{\text {tmax-mono }}$.

The differences between the monomeric and the dimeric system could be interpreted by assuming that a dimeric molecule is a monomeric with four times the potential $\epsilon$. In order to test if this would be the case, we rescaled pressure and temperature, $T^{* *}=T^{*} / 4$ and $P^{* *}=P^{*} / 4$, and replot the diagram illustrated in Fig. 3(a) obtaining Fig. 3(c). Even in this case the dumbbell system shows remarkable differences when compared with the monomeric case.

Comparing the monomeric and the dimeric versions of the system of particles interacting with the shoulder potential, we could observe that the addition of anisotropy changes the phase diagram of the fluid phase, being the differences significantly larger regarding the regions of the diffusion and structural anomalies. A proper choice of parameters, maybe a fine tuning of the distance $\lambda$ between the two particles of the same dimer, could shift the region of anomalies to specific ranges in the phase diagram. This could allow a design of materials with tuned anomalous properties.

\section{CONCLUSION}

We have addressed the question if the addition of anisotropy in a core-softened potential would lead to a modification in its anomalies and phases. For that purpose we have

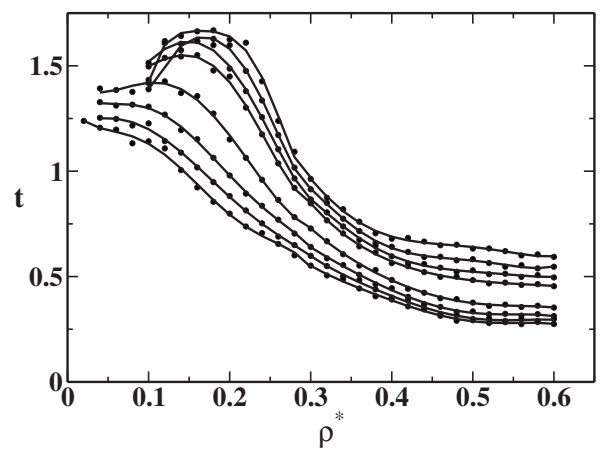

FIG. 5. Translational order parameter for the dumbbells system against density for fixed temperatures, which are $T^{*}=0.7,0.8,0.9,1.0,1.5,2.0,2.5$, and 3.0 from top to bottom. 
investigated a dimeric version of a previously studied monomeric model whose particles were subject to a core-softened potential interaction. ${ }^{18,19}$

We have found a much richer phase diagram for the dimers than that one obtained with the monomers. While for the monomeric system the solid phase is at temperatures much lower than the temperatures of the TMD region, for the dumbbell case the solid phase occupies a much wider region in temperatures and terminates at the edge of the TMD region. This indicates that the anisotropy favors certain ordering of the system at low temperatures and low or intermediate pressures. Regarding the enhancement in the pressuretemperature region occupied by the solid phase in the dimeric system, the same behavior is also observed in Lennard-Jones particles. ${ }^{55,56}$

In the present case two regions of solid phases separated by a fluid and a LCL region were observed, suggesting that the two length scales together with the anisotropic arrangement result in two solid phase densities. Also the presence of the LCL phase should be attributed to the dimers since this is not observed in the monomeric case. Even more surprising a very high pressure a liquid phase is also observed.

The thermodynamic, dynamic, and structural anomalous regions maintain the same hierarchy in the dimeric case as observed in the monomeric system. The structural anomalous region occupies a wider region in the pressure-temperature phase diagram when compared with the diffusion anomalous region and this region occupies a wider region when compared with the density anomalous region. This hierarchy is also observed in water. ${ }^{30,63}$ The dumbbell system, however, has pressures and temperatures in these anomalous regions much larger than the ones observed in the monomeric case. This behavior could be in principle explained mapping the dumbbell in a monomer with four times the potential interaction keeping the same interparticle distance. Since pressure and temperature scale with the interparticle potential, increasing the potential would lead to an increase in pressure and temperature.

The addition of an anisotropy in a core-softened potential leads to a richer phase diagram, but does not eliminate the thermodynamic, dynamic, and structural anomalies present in the isotropic system, but enlarges the solid state region. This result indicates that the use of dimers, trimers of even polymers of particles interacting with this isotropic potentials is a promising way to design complex molecules which might lead to systems with the same anomalies present in water or even other kinds of anomalous behavior.

\section{ACKNOWLEDGMENTS}

This work is partially supported by CNPq, INCT-FCx. We thank Evy Salcedo for helping with the analysis of the films of the liquid crystal phase.

${ }^{1}$ W. P. Krekelberg, J. Mittal, V. Ganesan, and M. Truskett, Phys. Rev. E 77, 041201 (2008).

${ }^{2}$ A. Scala, M. R. Sadr-Lahijany, N. Giovambattista, S. V. Buldyrev, and H. E. Stanley, J. Stat. Phys. 100, 97 (2000).

${ }^{3}$ G. Franzese, G. Malescio, A. Skibinsky, S. V. Buldyrev, and H. E. Stanley, Nature (London) 409, 692 (2001).
${ }^{4}$ S. V. Buldyrev, G. Franzese, N. Giovambattista, G. Malescio, M. R. Sadr-Lahijany, A. Scala, A. Skibinsky, and H. E. Stanley, Physica A 304, 23 (2002).

${ }^{5}$ S. V. Buldyrev and H. E. Stanley, Physica A 330, 124 (2003).

${ }^{6}$ A. Skibinsky, S. V. Buldyrev, G. Franzese, G. Malescio, and H. E. Stanley, Phys. Rev. E 69, 061206 (2004).

${ }^{7}$ G. Franzese, G. Malescio, A. Skibinsky, S. V. Buldyrev, and H. E. Stanley, Phys. Rev. E 66, 051206 (2002).

${ }^{8}$ A. Balladares and M. C. Barbosa, J. Phys.: Condens. Matter 16, 8811 (2004).

${ }^{9}$ A. B. de Oliveira and M. C. Barbosa, J. Phys.: Condens. Matter 17, 399 (2005).

${ }^{10}$ V. B. Henriques and M. C. Barbosa, Phys. Rev. E 71, 031504 (2005).

${ }^{11}$ V. B. Henriques, N. Guissoni, M. A. Barbosa, M. Thielo, and M. C. Barbosa, Mol. Phys. 103, 3001 (2005).

${ }^{12}$ P. C. Hemmer and G. Stell, Phys. Rev. Lett. 24, 1284 (1970).

${ }^{13}$ E. A. Jagla, Phys. Rev. E 58, 1478 (1998).

${ }^{14}$ N. B. Wilding and J. E. Magee, Phys. Rev. E 66, 031509 (2002).

${ }^{15}$ R. Kurita and H. Tanaka, Science 306, 845 (2004).

${ }^{16}$ L. Xu, P. Kumar, S. V. Buldyrev, S.-H. Chen, P. Poole, F. Sciortino, and H. E. Stanley, Proc. Natl. Acad. Sci. U.S.A. 102, 16558 (2005).

${ }^{17}$ P. A. Netz, J. F. Raymundi, A. S. Camera, and M. C. Barbosa, Physica A 342, 48 (2004).

${ }^{18}$ A. B. de Oliveira, P. A. Netz, T. Colla, and M. C. Barbosa, J. Chem. Phys. 124, 084505 (2006).

${ }^{19}$ A. B. de Oliveira, P. A. Netz, T. Colla, and M. C. Barbosa, J. Chem. Phys. 125, 124503 (2006).

${ }^{20}$ A. B. de Oliveira, M. C. Barbosa, and P. A. Netz, Physica A 386, 744 (2007).

${ }^{21}$ A. B. de Oliveira, P. A. Netz, and M. C. Barbosa, Eur. Phys. J. B 64, 481 (2008).

${ }^{22}$ A. B. de Oliveira, G. Franzese, P. A. Netz, and M. C. Barbosa, J. Chem. Phys. 128, 064901 (2008).

${ }^{23}$ A. B. de Oliveira, P. A. Netz, and M. C. Barbosa, Europhys. Lett. 85, 36001 (2009).

${ }^{24}$ Yu. D. Fomin, N. V. Gribova, V. N. Ryzhov, S. M. Stishov, and D. Frenkel, J. Chem. Phys. 129, 064512 (2008).

${ }^{25}$ N. G. Almarza, J. A. Capitan, J. A. Cuesta, and E. Lomba, J. Chem. Phys. 131, 124506 (2009).

${ }^{26}$ M. Pretti and C. Buzano, J. Chem. Phys. 121, 11856 (2004).

${ }^{27}$ H. M. Gibson and N. B. Wilding, Phys. Rev. E 73, 061507 (2006).

${ }^{28}$ S. Sastry, P. G. Debenedetti, F. Sciortino, and H. E. Stanley, Phys. Rev. E 53, 6144 (1996).

${ }^{29}$ O. Mishima and H. E. Stanley, Nature (London) 396, 329 (1998).

${ }^{30}$ P. A. Netz, F. W. Starr, H. E. Stanley, and M. C. Barbosa, J. Chem. Phys. 115, 344 (2001).

${ }^{31}$ J. R. Errington and P. D. Debenedetti, Nature (London) 409, 318 (2001).

${ }^{32}$ P. A. Netz, F. W. Starr, M. C. Barbosa, and H. E. Stanley, J. Phys. B 101, 159 (2002).

${ }^{33}$ H. Thurn and J. Ruska, J. Non-Cryst. Solids 22, 331 (1976).

${ }^{34}$ T. Tsuchiya, J. Phys. Soc. Jpn. 60, 227 (1991).

${ }^{35}$ G. E. Sauer and L. B. Borst, Science 158, 1567 (1967).

${ }^{36}$ S. J. Kennedy and J. C. Wheeler, J. Chem. Phys. 78, 1523 (1983).

${ }^{37}$ C. A. Angell and H. Kanno, Science 1121, 193 (1976).

${ }^{38}$ C. A. Angell, R. D. Bressel, M. Hemmatti, E. J. Sare, and J. C. Tucker, Phys. Chem. Chem. Phys. 2, 1559 (2000).

${ }^{39}$ R. Sharma, S. N. Chakraborty, and C. Chakravarty, J. Chem. Phys. 125, 204501 (2006).

${ }^{40}$ M. S. Shell, P. G. Debenedetti, and A. Z. Panagiotopoulos, Phys. Rev. E 66, 011202 (2002).

${ }^{41}$ P. H. Poole, M. Hemmati, and C. A. Angell, Phys. Rev. Lett. 79, 2281 (1997).

${ }^{42}$ S. Sastry and C. A. Angell, Nature Mater. 2, 739 (2003).

${ }^{43}$ P. C. Hemmer, E. Velasco, L. Medeiros, G. Navascués, and G. Stell, J. Chem. Phys. 114, 2268 (2001).

${ }^{44}$ M. Agarwal, R. Sharma, and C. Chakravarty, J. Chem. Phys. 127, 164502 (2007).

${ }^{45}$ C. H. Cho, S. Singh, and G. W. Robinson, Faraday Discuss. 103, 19 (1996).

${ }^{46}$ S. A. Egorov, J. Chem. Phys. 128, 174503 (2008).

${ }^{47}$ S. A. Egorov, J. Chem. Phys. 129, 024514 (2008).

${ }^{48}$ S. Zhou, Phys. Rev. E 74, 031119 (2006).

${ }^{49}$ S. Zhou, Phys. Rev. E 77, 041110 (2008).

${ }^{50}$ S. Zhou, J. Chem. Phys. 130, 054103 (2009). 
${ }^{51}$ L. Xu, S. Buldyrev, C. A. Angell, and H. E. Stanley, Phys. Rev. E 74, 031108 (2006).

${ }^{52}$ Z. Yan, S. V. Buldyrev, N. Giovambattista, and H. E. Stanley, Phys. Rev. Lett. 95, 130604 (2005).

${ }^{53}$ Z. Yan, S. V. Buldyrev, N. Giovambattista, P. G. Debenedetti, and H. E. Stanley, Phys. Rev. E 73, 051204 (2006).

${ }^{54}$ G. Franzese, J. Mol. Liq. 136, 267 (2007).

${ }^{55}$ C. Kriebel and J. Winkelmann, J. Chem. Phys. 105, 9316 (1996).

${ }^{56}$ C. Vega, C. McBride, and E. de Mibuel, F. J. Blas, and A. Galindo, J. Chem. Phys. 118, 10696 (2003).

${ }^{57}$ S. J. Plimpton, J. Comput. Phys. 117, 1 (1995).
${ }^{58}$ H. J. C. Berendsen, J. P. M. Postuma, W. F. van Gunsteren, A. DiNola, and J. R. Haak, J. Chem. Phys. 81, 3684 (1984).

${ }^{59}$ J. P. Ryckaert, G. Ciccotti, and H. J. C. Berendsen, J. Comput. Phys. 23, 327 (1977).

${ }^{60}$ G. Malescio and G. Pellicane, Nature Mater. 2, 97 (2003).

${ }^{61}$ G. J. Pauschenwein and G. Kahl, J. Chem. Phys. 129, 174107 (2008).

${ }^{62}$ P. Kumar, G. Franzese, and H. E. Stanley, Phys. Rev. E 73, 041505 (2006).

${ }^{63}$ P. A. Netz, F. W. Starr, M. C. Barbosa, and H. E. Stanley, Physica A 314, 470 (2002). 